XII.

\title{
Über Syringomyelie.
}

Von

Dr. Franz C. Müiler,

Nervenarzt in München.

Seit der Finführung der Unfallgesetzgebung haben sich die Ansichten der Arzte auf einem Gebiete wesentlich geändert, nämlich auf dem der Ätiologie der Nervenkrankheiten. Mehr wie man als schlimmster Pessimist voraussetzen konnte, trat das Trauma in den Vordergrund und wir sehen heute die schwersten Neurosen und Psychosen sich an relativ unbedeutende Unfälle anschliessen; Unfälle, die in der Periode vor dem Inkrafttreten der Unfallgesetze in wenigen Wochen und ohne spätere Folgen ausheilten, werden heute die Crux medicorum, weil sie allen Kurversuchen Trotz bieten. Am bekanntesten wurde die "traumatische Neurose", deren Vater Oppenheim wohl oft schon mit Goethes Zauberlehrling ausrief: „Die ich rigf, die Geister, werd” ich nun nicht los." Wir kennen eine unirerselle und eine partielle traumatische Neurose und sehen staunend, dass zum Zustandekommen dieser dunkelsten aller Neurosen oft schon ein geringfügiger psychischer Shock genügt, der bei einem Nichtarbeiter in einer Stunde spätestens vergessen ist oder überhaupt nicht auftritt. Wohl kaum einer der in Unfalluntersuchungen erfahrenen Kollegen wird seine Verwunderung bemeistern können, wenn er sieht, wie die verschiedenen Stände verschiedenartig auf traumatische Einflüsse reagieren; der Student quittiert einen Säbelhieb, der tief in den Knochen eingedrungen ist oder einen "Flachen“, der ihn fast umgeworfen hätte, mit dem normalen Heilverlauf, der - nicht versicherte - Techniker arbeitet stundenlang unter ständiger Lebensgefahr bei einem Brückeneinsturz oder in einer brennenden Pulverfabrik. Von seinen Befehlen hängt das Leben von Dutzenden von Menschen ab; trotz dieser schweren Verantwortung und des ihm selbst drohenden Todes kommt er über die Katastrophe mit einer reparablen Nervenerregung weg, wie auch der Offizier nach den moralischen Eindrücken einer modernen Schlacht noch lange nicht an traumatischer Neurose erkrankt. Aber der 
Arbeiter, dem ein Stückchen Stein auf den durch einen Filzhut geschützten Kopf fiel, ohne dass ein Haar gekrümmt wurde, verliert den an den Unfall sich anschliessenden Kopfschmerz und Schwindel nicht und bleibt für Jahre, mitunter für sein Leben arbeitsunfähig. Ihm erzeugt derselbe Stein, der zufällig nicht den Kopf, sondern den Oberschenkel getroffen hat, eine blutunterlaufene Stelle und im Anschluss an den bei der Affäre ausgestandenen Schrecken (der ihn nicht gehindert hatte, noch Tage lang fortzuarbeiten) eine partielle traumatische Neurose der betreffenden Extremität. Alle unsere Kenntnisse von der Widerstandsfähigkeit des zentralen und peripheren Nerrensystems werden auf den Kopf gestellt, wenn wir den Unterschied in der Reaktion der einzelnen Stände sehen und konstatieren müssen, dass der im Freien aufgewachsene, robuste, körperlich kerngesunde Arbeiter empfindlicher ist, wie der Stubengelehrte oder — der Nichtversicherte.

Strümpell hat seinerzeit einen Lichtstrahl in das herrschende Iunkel geworfen, indem er uns zeigte, dass die "Begehrungsvorstellungen“ der versicherten Arbeiter eine Art ron Hysterie erzeugen, die eben als solche unser ärztliches Urteil auf eine schwere Probe stellt, denn lieber zehnmal einem Unwürdigen eine Rente begutachten als einmal einem wirklich Kranken Unrecht tun! - -

Als Historiker sehe ich seit langem meine Wissenschaft und deren Ausübung vielleicht mit anderen Augen an, als mancher Fachkollege. Ich sehe zweierlei: 1. dass man immer mehr den Begriff der traumatischen Neurose einzudämmen versucht und an Stelle derselben die sogenannte traumatische Hysterie setzt und 2. dass eine Reihe von Krankheiten in der Unfallbegutachtung mehr und mehr in den Vordergrund tritt, die man früher nur äusserst selten konstatieren konnte. Zu diesen zählt die Syringomyelie.

Bevor ich aber näher auf dieselbe eingehe, sei es dem Historiker vergönnt, mit wenigen Worten zu betonen, dass die Neurasthenie anscheinend abgewirtschaftet hat. Schon vor 12 Jahren konnte ich in meinem "Handbuch der Neurasthenie“ die Hoffnung aussprechen, dass unsere moderne Kultur nicht immer mit siebenmeilenstiefeln vorwärts schreiten wird und mit dem Rïckschlag, der sicher eintreten muss, auch seltener werden wird. Der Rückschlag ist gekommen: in der Kunst, in der Literatur, in der Politik und auch in der Wissenschaft! Wir zehren an den Resten eines reichen Mahles, das unseren Vütern aufgetischt worden war, die Menschheit bekam Zeit, in die neuen Verhältnisse hineinzuwachsen und sich von den Schädlichkeiten dieser stürmischen Jahre zu erholen. Die Neurasthenie, als signum einer Kulturepoche, wird seltener, je mehr sich diese Kultur erschöpft hat. An Stelle der industriellen, wirtschaftlichen und wissenschaftlichen Elevation trat eine Stagnation, die wir mit sportlicher Ablenkung ausfüllen, weil wir instinktiv fühlen, dass dem Cerebrum zuviel zugemutet wurde und die Folgen dieser Überanstrengung auf geistigem Gebiete nur durch körperliche Ablenkung paralysiert werden können. Aber wie der Mensch niemals sofort die goldene Mitte findet, sondern zweifelnd zuerst nach rechts und links abirrt, so hat auch der Sport seine Jünger aus den ungefährlichen Bahnen hinaus- 
geworfen und durch Überanstrengungen Sch ̈̈dlichkeiten erzeugt, die nun nicht das Gehirn treffen, sondern die Zirkulationsorgane. Es ist von allergrösstem Interesse zu sehen, wie die Herzkrankheiten an Häufigkeit zunebmen und wie die neuen Herzuntersuchungs- und Behandlungsmethoden, die auf die funktionelle Dilatation Rücksicht nehmen, rasche Anerkennung gefunden haben.

Kehren wir zur traumatischen Neurose zurück, so fallen uns bei kritischer Sichtung eines grossen Materiales verschiedene Tatsachen auf, welche die Kritik herausfordern: In einzelnen Gegenden Deutschlands, welche gerade zu den industrieilsten gehören, sind Fälle von traumatischer Neurose so selten, wie heute noch bei uns die Syringomyelie, in anderen Arbeitszentren häufen sich die Fälle, ja man kann sogar behaupten, dass in gewissen Stadtteilen drei- und viermal so viele Neurosen vorkommen, als in anderen gleich stark von Arbeitern bewohnten. Ferner kann es dreist ausgesprochen werden, dass die Schwere der Symptome häufig mit der Zahl der Kontrolluntersuchungen zunimmt; und nach einem Aufenthalt in Sanatorien und Krankenhäusern oder in Privatkliniken steht das Krankheitsbild meist in voller Vollendung vor uns.

Wir sind weit daron entfernt, damit den Kollegen einen Vorwurf zu machen, aber wenn sich ein solcher aus unseren Darlegungen abstrahieren lässt, so gilt er den Verhältnissen, in denen wir gross geworden sind und arbeiten müssen. - Genau dieselben Beobachtungen, die wir bei Arbeitern machen, die den Berufsgenossenschaften zur Last fallen, drängen sich uns bei den Privatversicherungen auf, deren Schutzbefohlene den besseren und besten Kreisen angebören. In puncto Unfall sind sich alle Menschen leider! gleich; bei allen dieselben Begehrungsvorstellungen, bei allen die gleiche (hysterische) Sucht, Krankheitssymptome zu erfinden oder zu übertreiben und längst vorhandene Leiden auf den letzten Unfall zurückzubeziehen. Einer der Hauptfehler, der in dieser Hinsicht gemacht wird, liegt darin, dass die ärztlichen Atteste in ihrer Vollständigkeit den Verletzten mitgeteilt werden. Was geht es im Grunde den Verletzten an, dass sein Patellarreflex gesteigert ist -eine Anomalie, die man auch in der Privatsprechstunde weislich für sich behält? Wenn aber der Kranke aufmerksam gemacht wird, dass die traumatische Neurose sich möglichenfalls in gesteigerten Retlexen zeigt, so simuliert er einen Reflex, bevor noch die untersuchende Hand das Ligamentum getroffen hat. Und wenn gar der Verletzte die Bedeutung des Dynamometers versteht, so scheut er sich nicht, nur einen Ausschlag von 5 oder $10 \mathrm{~kg}$ zu leisten, wo er 60 und $80 \mathrm{~kg}$ leisten könnte - und merkt nicht, wie man die fingierte Kraftanstrengung doch richtig eintaxieren kann. Je öfter die Unfallkranken untersucht werden, um so grössere Routine erwerben sie, und war gar einer längere Zeit auf der Hochschule -- im Krankenhaus, wo er Gelegenheit hatte, im Verkehr mit Leidensgefährten sich fortzubilden, so erzählt er ohne Stocken ein Krankheitsbild (Schwindel, Kopfdruck, Herzklopfen, Schmerzen im Rücken etc.), das den unerfahrenen Untersucher regelmässig täuscht.-- Bei häufiger Gelegenheit, Unfallverletzte zu untersuchen, fahndet man gewissermassen nach objektiven Symptomen und atmet auf, wenn man eine Dermo- 
graphie, eine Pupillendifferenz, ein Zittern der geschlossenen Augendeckel gefunden hat, obgleich man sich im Innern und vor dem wissenschaftlichen Gewissen sagen muss, dass damit noch lange nichts bewiesen ist. Aber man will die Beschwerden erklären und klammert sich in der Not an die fernsten Symptome, muss man ja oft genug auch ein non liquet schreiben, wo man absolut nichts gefunden hat. -

Mir scheint, als ob in der Beurteilung der Unfallkranken, die ja noch eine junge, nichts weniger als fixierte Wissenschaft ist, viel zu konnivent vorgegangen würde. Daran sind verschiedene Faktoren schuld: die Unsicherheit des Untersuchenden, der sich oft Symptomen gegenüber sieht, die er in kein landläufiges Schema einreihen kann, das Mitleid mit dem Kranken, welcher doch nicht obne Grund so lange arbeitslos herumsitzt und eine gewisse Scheu vor der Öffentlichkeit. Welchem gesuchten Unfallbegutachter ist es nicht schon begegnet, dass er in sozialdemokratischen und anderen Blättern heruntergerissen wurde - mit Entstellung der Tatsachen! Die grosse Arbeit, die mitunter schon an einer Untersuchung hängt, wird pro nihilo geschätzt, geschätzt wird nur der klingende Erfolg -- die Rente. Und hat ein Arzt nach langen Gewissenskämpfen in einem konkreten Falle das schwerwiegende Wort „Simulant" gesprochen, so zetert die Presse, das Sprachrohr der unterdrückten Arbeiter, die fast ohne Ausnahme die Versicherung als eine Pensionskasse ansehen. - Die Unfallbegutachter müssen unabhängige Männer sein, unabhängig nach oben und nach unten. Sie sind es auch zumeist, wenigstens in den grossen Städten, aber in einzelnen Bezirken hängt der Arzt so sehr von gewissen Faktoren ab, über die er wegschauen müsste, dass ein unbefangenes Urteil nahezu eine Selbstverleugnung oder ein Selbstmordversuch ist. - Wir bleiben immer Menschen; ich möchte den kennen, der in der Unfallbegutachtung eines reichen Fabrikherrn, von dessen Wort die Fabrikkasse abhängt, so ruhig urteilte, wie er es als klinischer Assistent bei einem Falle tun kann, der ihn später nicht weiter interessiert! -

Es ist auch ein prinzipieller Fehler, dass die jungen, leider auch die alten Ärzte die Versicherungsgesetze nicht in dem wünschenswerten Masse kennen. Mit der Einführung der neuen Gesetze erwuchs uns eine neue Aufgabe, der wir nur gerecht werden können, wenn wir das Gesetz kennen, so gut, wie es der Jurist kennen muss. Dazu gehört die richtige Abschätzung des Schadenfalles. Wenn ich lese, dass für eine $2 \mathrm{~cm}$ lange Hautwunde am Kopfe ohne jede Komplikation $100 \%$ bewilligt werden und zwar für 14 Tage und 3 Wochen, so sehne ich mich als Unfallbegutachter nach der Zeit zurück, wo in solchen Fällen der Bader einfach ein Pflaster aufdrückte und - der Fall erledigt war. Auch ist es direkt eine Unterstützung der „Begehrungsvorstellungen“, wenn man bei einfachen Verletzungen, sagen wir bei einer Distorsion des Fussgelenkes, bis zum Schlusse die ganze Erwerbsfähigkeit ausschaltet. Man wird nicht iber Nacht gesund! Wie der "Distorquierte", wenn er nicht versichert ist, einige Tage mit dem Eisbeutel auf dem Sopha liegt, dann im Zimmer herumhumpelt, endlich eine, zwei Stunden ausgeht und allmählich gesundet, ebenso ist es beim Versicherten, der auch denselben Hei- 
lungsverlauf bietet und schon lange arbeiten könnte, wenn er "wollte". - Von den anatomischen und physikalischen Fehlern der Gutachten möchte ich aus kollegialen Gründen Abstand nehmen. Wer das "Os pisiforme" am Fusse fühlt, wer bei einem Einäugigen Doppelbilder beschreibt, der zieht am klügsten sein Schild als Vertreter unserer Wissenschaft ein. -

Ich bin vom Thema abgekommen! Denn ich wollte von der Syringomyelie sprechen und habe mich in allgemeine Erörterungen eingelassen. Ich konnte schon betonen, dass genannte Krankheit mehr und mehr bekannt wird und in gewissem Sinne im Begriffe steht, die etwas diskreditierte traumatische Neurose zu ersetzen. Die Zahl der Fälle von Syringomyelie mehrt sich zusehends und ebenso die Monographien iiber dieselbe. Schon 1564 beschrieb Etienne eine Höhlung in der inneren Substanz des Rückenmarkes, ihm folgte fast 200 Jahre später Morgagni. Alle späteren Arbeiten sind nur Diagnosen nach der Obduktion, bis Kahler und Schultze 1882 den Nachweis erbrachten, dass die Krankheit auch in vivo erkannt werden kann. Schon 1887 referierte Anna Bäumler über 112 anatomische und klinische Fälle. Nunmehr folgen die Publikationen rascher, so dass zur Zeit eine reiche Literatur vorliegt.

Finen grossen Fortschritt bedeuten die Untersuchungen Hoffmanns, der die Höhlenbildung auf entwickelungsgeschichtliche Anomalien und auf Gliawucherungen zurückführt. In Frankreich weisen die Forscher fast einstimmig auf die Beziehungen der Syringomyelie zur Lepra und zur Morvanschen Krankheit hin. Das grösste und umfassendste Werk gab uns Hermann $\mathrm{Schlesinger}$ in seiner bekannten Monographie.

Wir setzen die häufigeren Symptome des vielgestaltigen Krankheitsbildes als bekannt voraus und haben an dieser Stelle nur die Absicht, den Einfluss traumatischer Einflüsse nälıer zu studieren. Dass man bei der äusserst dunklen Ätiologie auch an das Trauma dachte, kann nicht überraschen und ist einerseits auf den Forschungseifer der untersuchenden Ärzte, andererseits aber auch auf das Entgegenkommen der Patienten zurückzuführen, die, soweit es sich um Arbeiter handelt, ja den verschiedenartigsten Verletzungen ausgesetzt gewesen waren, von denen gegebenenfalls die eine oder andere ätiologische Bedeutung haben konnte.

Wenn die Wirbelsäule ein schweres Trauma erleidet, kann das Mark makroskopisch vollständig unversehrt aussehen. Trotzdem kann das Gewebe der weissen, noch mehr der grauen Substanz schwere Schädigungen erfahren haben, die durch Blutgefässzerreissungen zu langgestreckten Blutungen und sekundüren Erweichungen führen können. Nach einiger Zeit wird der zerstörte, nicht mehr funktionsfähige, als Fremdkörper wirkende, Teil der Rückenmarkssubstanz resorbiert und das gesund gebliebene Mark durch eine Bindegewebs-Gliaproliferation differenziert. Wir haben dementsprechend auch klinisch das Bild anfangs sehr schwerer Symptome, die sich langsam verlieren, aber einen mehr weniger grossen Funktionsdefekt zurücklassen. Dies ist aber nicht der Verlauf der typischen Syringomyelie, die fortschreitend degeneriert, sondern muss vielmehr als ein langsamer Ausheilungsprozess einer Hämatomyelie oder 
einer traumatischen Nekrose aufgefasst werden. Mithin muss im allgemeinen die cystische Vernarbung einer traumatisch erzeugten Blutung resp. Erweichung der Rückenmarkssubstanz scharf von der genuinen Syringomyelie getrennt werden, was mit der bei einzelnen Autoren vorkommenden Bezeichnung "Pseudo-Syringomyelie“ auch deutlich ausgedriickt ist. Nur in ganz seltenen Fällen kann, wie Minor annimmt, die Gliaproliferation der Cystenwand den Ausgangspunkt für eine progressive Gliose abgeben, doch ist dieser Modus nach Schlesinger als äusserst selten, jedenfalls nicht als sichergestellt $\mathrm{zu}$ bezeichnen.

Anders liegen die Dinge, wenn sich nach einem Trauma nicht sofort dıe bekannten Symptome der Syringomyelie gezeigt haben, sondern erst nach einem längeren oder kürzeren Latenzstadium in die Erscheinung treten. Es sind dies die für den Unfalluntersuchenden wichtigen Fälle. Da man nicht oder nur durch Zufall in die Lage kommt, einen gesunden Arbeiter zu untersuchen, so kann man sich nicht darüber klar werden, ob nicht doch schon vor dem inkrimierten Unfalle Symptome vorhanden waren, welche der Fachmann als beginnende Syringomyelie gedeutet hätte, welche aber die Arbeitsfähigkeit noch nicht in Frage gestellt hatten und deshalb übersehen werden konnten.

Dagegen ist nicht zu bestreiten, dass eine schleichende Syringomyelie durch ein das Rückenmark treffendes Trauma, und sei dies auch nur eine einfache Erschütterung, progredienter gemacht wird, dass also nach dem Unfall das vor demselben schon kranke Individuum rasch der Arbeitsunfähigkeit anheimfällt. Diesen Standpunkt nimmt anch Schlesinger ein, der die Möglichkeit des Zusammenhanges betont.

Ein wiederholt gemachter Versuch, die Entstehung der Syringomyelie von einer ascendiejenden Neuritis abhängig zu machen, ist nach eingehender klinischer Musterung der gebotenen Symptome bei dem Mangel beweisender Obduktionsbefunde von der Hand zu weisen.

Westphal hat in jüngster Zeit zwei Fälle veröffentlicht, in welchen auch toxische Einwirkungen (Alkohol) herangezogen wurden. Es dreht sich nach ihm die Frage darum, ob einfache „molekuläre Erschütterungen“, Erschütterungen mit Blutungen und mit toxischen Einflüssen einhergehende oder auf toxischen Einflïssen beruhende Blutungen im stande sind, die progressiv-irritativen Gewebsveränderungen der Syringomyelie zu erzeugen. Er kommt zu dem Schlusse, dass dies wahrscheinlich ist, aber dass die Entstehung der progressiven Gliose noch ebenso dunkel ist wie die Entstehung der Geschwülste überhaupt. Huismanns, dem wir den jüngsten Beitrag zum vorliegenden Thema verdanken, deponiert, dass das Bild der Syringomyelie durch Gliose oder durch die sekundäre Infektion einer Rückenmarksblutung hervorgerufen werden kann; meist aber dreht es sich um eine chronisch fortschreitende, in Erweichung übergehende infektiöse Myelitis im Anschluss an Embolie oder 'Thrombose der zentralen Gefässe des Rückenmarks. Wir müssen das Trauma als Krankheitsursache, nach seiner Meinung, anerkennen, wonn es einen vorher "Gesunden" getroflen hat. 
Schlesinger formuliert seine Ansichten kurz dahin: „Die Syringomyelie scheint bisweilen kongenital zu sein, öfters aber dürfte nur eine kongenitale Anlage vorhanden sein, welche durch Schädlichkeiten mannigfacher Art (Infektionskrankheiten, Gravidität, Temperatureinflüsse), namentlich aber durch Traumen zur Weiterentwickelung gebracht wird. Öfter dürften mehrere ätiologische Momente miteinander konkurrieren. Ein strikter Beweis ist aber hierfür bisher durch die klinische Beobachtung nicht beigebracht, ebensowenig aber auch für die Annahme, dass diese Höhlenbildungen infolge der eben aufgezählten Einflüsse in einem ganz normalen Rückenmark entstehen können."

Ich komme nun zur Schilderung meiner Fälle:

S. R., 36 Jahre alt, lediger Wegmacher aus Oberbayern, stammt aus gesunder Familie; war als Kind immer gesund. Mit 30 Jahren Lungenentzïndung. Potus und Syphilis negiert. Mit 33 Jahren akquirierte er ein Geschwür an der rechten Hand, welches eine "Blutvergiftung" nach sich zog und mehrfache Operationen nötig machte. Mit 35 Jahren Nagelgeschwür an der rechten Hand, welches ein Panaritium und eine Beugekontraktur des Mittelfingers verursachte. Langsam entwickelte sich eine Bengekontraktur der ibrigen Finger der rechten Hand. Im letzten Winter erfror sich R. die linke Hand und wegen Gangrän des linken Mittelfingers erfolgte Amputation desselben, worauf sich auch links Kontrakturen einstellten. -

Hat zur Zeit der Untersuchung starke Schmerzen in der linken Hand und im linken Fuss, "wie wenn Feuer und Ameisen" darin wären. Beim Stehen fühlt er Zittern in den Füssen. Absolute Impotenz und Alkoholintoleranz. Die Arbeitsfühigkeit ist bei dem \%ustand der Hände auf ein Minimum reduziert. Einen' Unfall hat er nicht erlitten, wenn die Blutvergiftung vor drei Jahren nicht als Unfall aufzufassen ist. Interessant ist, dass R. wegen Skoliose der Wirbelsäule militärfrei geworden ist. -

Die Hände sind stark vergrössert und geschwollen. Vom Zeige- und Mittelfinger der rechten Hand fehlt die vorderste Phalanx. An der linken Hand fehlt der ganze Mittelfinger, der Zeigefinger ist cyanotisch verfärbt, die anderen erhalten gebliebenen Fïnger sind rot verfärbt, fühlen sich kalt an und zeigen insgesamt starke Beugekontraktur, so dass beide Hände zur Faust geballt sind. Starker, kleinschlägiger Tremor. Zittern des ganzen Oberkörpers. Die Sensibilität im Gebiete des linken Zeigefingers ist völlig erloschen, an den iibrigen Fingern hochgradig herabgesetzt. Schmerzempfindung allenthalben stark vermindert, Schmerzleitung verlangsamt. Die Tastkreise sind rechts erweitert, links nicht zu bestimmen, weil Patient iiberhaupt nichts emptindet. -

Pupillen mittel- und gleichweit, reagieren langsam, aber gleich.

Zunge zittert. Beim Stehen mit geschlossenen Augen starkes Zittern der Augendeckel und Schwanken des Oberkörpers nach vor- und rückwärts; dabei wackelt der Kopf beständig. lich ist.

Mässige Kyphoskoliose der Wirbelsäule, die nirgends druckempfind- 
Bauchdecken- und Kremasterreflex erloschen. Würg- und Konjunktivalreflex sehr schwach. Patellarreflexe sehr stark, klonisch. Fussklonus links sehr stark, rechts angedeutet. Sensibilitätsstörungen der unteren Extremitäten sind nicht zu finden.

Skandierende Sprache.

Breitspuriger Gang. Herabgesetzte Intelligenz.

Herz nicht vergrössert, Töne rein, regelmässig, sehr beschleunigt, 152 pro Minute. Ernährungsstand unternormal. Deutliche Anämie.

Der Mann wusste von seiner zentralen Erkrankung nichts. Fr wurde mir zugeschickt mit der Diagnose: "Geschwüre an den Händen" und wollte eine Invalidenrente erhalten. Zu irgendwelcher Simulation oder.Übertreibung hatte der Untersuchte keine Veranlassung. Fs besteht kein Zweifel, dass es sich im vorliegenden Falle um eine typische Syringomyelie handelt, sowie dass die verschiedenen Affektionen an den Händen nur deshalb die bezeichneten Folgen haben konnten, weil die Empfindungsfähigkeit des R. von vornherein herabgesetzt war. Eine traumatische Läsion des Rückenmarkes hat zweifellos nie stattgefunden, wohl aber deutet die Militärbefreiung wegen Skoliose darauf hin, dass schon damals der Beginn des Leidens deklariert war. Es ist dies zwar nicht bewiesen, weil ja jeder Gesunde aus anderen Gründen auch skoliotisch werden kann, aber im Zusammenhalt mit den übrigen Symptomen ist es wabrscheinlich. - Der Fall hat aber noch eine andere Seite! R. wurde vielfach untersucht, auch zur Kur einer medico-mechanischen Anstalt übergeben. Erst als deren Arzt an den bisherigen Diagnosen Zweifel bekam, zog er den Neurologen zu, der dem Invaliden, nicht dem Unfallverletzten, zu seinem Rechte verhelfen konnte. -

Der 2. Fall hat deshalb grosses Interesse, weil er auf 12-15 Jahre zurückgeht. Er betrifft einen 34 Jahre alten, verheirateten Monteur aus Niederbayern, der schon zu Anfang der neunziger Jahre auffallende Empfindungsstörungen am rechten Arme zeigte, die „anfallsweise“ auftraten. N ach her erlitt er mehrere Cnfälle, von denen einer mit einer starken Commotio spinalis vergesellschaftet war. Im Anschluss daran zeigte sich bei Erhöhung der Patellarreflexe eine Empfindungsstörung des rechten Armes, die Ziemssen veranlasste, schon 1893 die Diagnose auf Syringomyelie zu stellen. Es wurde in den damaligen Gutachten betont, dass die Krankheit in ihrem Keime schon vor den Unfällen bestanden habe, aber durch dieselben so weit vorgeschritten wäre, dass die Erwerbsfähigkeit Schaden leiden musste. Auf Grund dieses Gutachtens wurde eine Unfallrente von anfänglich 50, dann $70 \%$ ausgesprochen. Schliesslich musste dieselbe auf $100 \%$ erhöht werden. Alle Begutachter - es waren deren nicht wenige - hatten über die Diagnose keinen Zweifel. Ab 1898 erholte sich der Rentenempfänger und heute bietet er von allen früheren Symptomen nichts mehr als Herabsetzung der Sensibilität am rechten Arme, die ihn gelegentlich veranlasst, obertlächliche, einmal auch eine tiefgreifende Verbrennung nicht zu empfinden und zu ignorieren. Ausserdem ist er ein krüftig gebanter Mann mit athletischer Muskulatur (Dynamometer $R=110$, normalen Reflexen, normaler Heratätigkeit. Potenz tadellos. 
Keine Intoleranz gegen Alkohol. Aussehen vorzüglich. Gang, Sprache, Psyche normal. Es könnte gut die jetzt bestehende Rente von $15 \%$ eingestellt werden, wenn nicht die Erfahrung lehrte, dass den in der Remission befindlichen Fällen von Syringomyelie nicht zu tranen ist. Dies veranlasste auch den Schreiber dieses, die Belassung der Rente zu begutachten, damit keinesfalls die Erwerbsverhältnisse des Untersuchten verändert werden. Ich bin dabei überzeugt, das es falsch wäre, von einer "mit Defekt geheilten Syringomyelie" zu sprechen, dass vielmehr in absehbarer Zeit der Gang auf der schiefen Ebene wieder beginnen wird.

Die Syringomyelie ist eine sehr seltene Krankheit. Sie in den Rahmen der Unfallkrankheiten aufzunehmen, besteht kein Grund. In den meisten Fällen dreht es sich um eine Steigerung von Symptomen, die schon vorher gegehen waren, aber in der latenz oder wegen der Geringfiigigkeit nicht gefühlt werden. Da die Versehlimmerung eines Leidens, falls ein Unfall vorliegt, auf den Unfall selbst gesetzlich zurückgeführt werden muss, so haben wir auch eine „traumatische Syringomyelie“, eine Diagnose, die aber bis zum Fortschreiten der Mikroskopie des Rückenmarkes und der klinischen Forschung als eine Liberalitätsdiagnose bezeichnet werden muss. 